\title{
Natural History of Radiological Changes on CT Chest from COVID-19 Infection Rehabilitation Patients
}

Ann Peng (D 819131357@qq.com)

The Affiliated Hospital of Nanjing University of Chinese Medicine

\section{Research}

Keywords: radiological changes, chest CT, rehabilitation, COVID-19

Posted Date: September 28th, 2021

DOI: https://doi.org/10.21203/rs.3.rs-923841/v1

License: (a) (i) This work is licensed under a Creative Commons Attribution 4.0 International License. Read Full License 


\section{Abstract}

Object囚 To investigate the chest CT manifestations of convalescent patients with COVID-19 in recovery phase.

Methods: 118 convalescent patients diagnosed as COVID-19 were followed up. According to their medical history, they were divided into 47 cases of severe disease group and 71 cases of mild disease group. Multi-slice spiral CT, high-resolution CT and pulmonary function were examined.

Results: 67 rehabilitated cases are normal on CT scan. The other CT findings were: (1) ground glass opacity lesions, including: localized patchy ground glass density lesions; Multi lobes and multi-stage ground glass opacities; Diffuse ground glass opacities lesions in both lungs. (2) The interlobular interstitium and interlobular septum were thickened. (3) Subpleural arc shadow/Subpleural lines. (4) Irregular fiber cord shadow/Irregular lines. (5) Tractive bronchiectasis. (6) Nodular consolidation of air space. (7) Cavitary lesions. No obvious mediastinal lymph node enlargement and pleural effusion were found. Pulmonary imaging and pulmonary function were improved after repeated reexamination. There was significant difference in CT findings and pulmonary function indexes between severe group and mild group $(\mathrm{P}<0.05)$.

Conclusion: The pulmonary manifestations of some convalescent patients with COVID-19 are basically normal. Ground glass density lesions are the main CT manifestations of convalescent patients, accompanied by varying degrees of pulmonary interstitial hyperplasia. The severe group had more severe pulmonary manifestations and poor pulmonary function than the mild group. With the extension of time, pulmonary lesions and pulmonary function gradually improved.

\section{Introduction}

Since the outbreak in Wuhan, China, in December 2019, Coronavirus Disease 2019 (COVID-19) has quickly spread worldwide; there are currently 228,206,384 confirmed cases of COVID-19 and 4,687,066 deaths in over 225 countries $^{1,2}$. The COVID-19 infection is associated with high rates of hospitalisation and mortality and this has placed healthcare systems under strain. As of 10 September 2021, there are 41,716,516 confirmed cases of COVID-19, and that of COVID-19-related deaths were 667,244 in United States of America, with the highest mortality rate reaching $2.16 \%{ }^{2} \varangle$ Based on the world total infection and

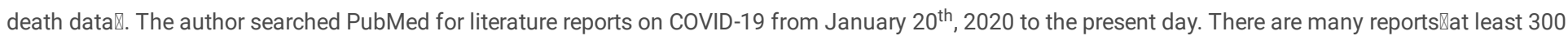
articles\on the non-contrast enhanced high-resolution computed tomography (HRCT) features of the lungs during the onset of COVID-19; however, few studies have described the radiological changes and outcome of residual lesions in the lungs of recovered patients ${ }^{3,4}$. Here, we review the HRCT features of 118 recovered COVID-19 patients at 22-465 days post-discharge. These features can be utilized to predict the prognosis and guide rehabilitation treatment of COVID-19

\section{Materials And Methods Study Subjects}

118 cases diagnosed as having COVID-19 between December 2019 and April 2021 were evaluated at the Xiangyang No.1 People's Hospital, Xiangyang (China). All of the patients had been enrolled in research protocols approved by Xiangyang No.1 People's Hospital Affiliated to Hubei University of Medicine. All of the COVID-19 patients provided written informed consent for this study.

The clinical data were collected from 118 recovered COVID-19 patients who were affected between December 2019 and April 2021. According to Clinical

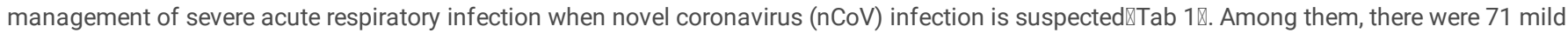
and 47 severe cases based on the World Health Organisation guidelines ${ }^{5,6}$. The patients were reexamined by $\mathrm{CT}$ at $22-465$ days post-discharge; the average time from discharge to the first reexamination was 31.3 days (22-36 days) $)$ the second reexamination was 60.5 days (43-88 days) $\square$ the third reexamination was 169.5 days (112-260 days) and the fourth reexamination was 352.6 days (195-465 days). There were 66 males and 52 females aged $20-71$ years old

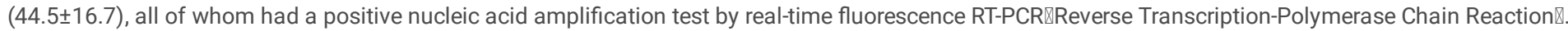
They conformed to the Clinical Diagnostic Criteria for COVID-19 released by the World Health Organization and China's Health Ministry. These 118 patients took chloroquine phosphate tablets ( $500 \mathrm{mg}$ BID for seven days for bodyweight $>50 \mathrm{~kg} ; 500 \mathrm{mg}$ BID on days 1 and 2, and $500 \mathrm{mg}$ QD from days 3 to 7 for bodyweight $₫ 50 \mathrm{~kg}$ ), Arbidol (Umifenovir囚Arbidol Hydrochloride Capsules) (adult $\ 200 \mathrm{mg}$ TID for no longer than 10 days), and high-flow nasal cannula $(\mathrm{HFNC})^{7}$. Discharge criteria were: CT scan showing absorption and improvement of the lesions; three negative nucleic acid tests (spaced 48 hours apart); and isolation for 14-21 days at designated hotels.

The following CT scanners: Toshiba Aquilion Prime Series 80-slice CT scanner and Siemens Definition AS 64-slice CT scanner were used to scan from the apices to the lung bases. CT images were acquired during a single breath hold $\nabla$ The main scanning parameters were as follows: tube voltage, $120 \mathrm{kVp}$; slice thickness, 1-10 mm; pitch, 0.9-1.4 mm; and field of view, $400 \mathrm{~mm} \times 400 \mathrm{~mm}$; matrix, 512×512; automatic tube current modulation; tube current, $100-400 \mathrm{mAs}$. All images were then reconstructed with a slice thickness of 0.5-1.4 mm with the same increment. The Minato AS-507 spirometer (Japan) was used to measure the FEV1.0\%(the percentage of forced expiratory volume in one second to the predicted volume ) for 118 recovered patients.

\section{Results}

1.Clinical follow-up:Upon the first and second reexamination, the cardinal symptoms were chest tightness in 21 cases, shortness of breath in 14 cases, fatigue in 6 cases, and joint pain in 3 case. While upon the third and above reexamination囚as radiological changes improved囚only 7 patients remain intermittent chest tightness. 
2.CT features upon the first reexamination: Among the 118 patients, HRCT revealed normal lungs in 24 recovered patients and residual lesions in the lungs of 94 patients: $\otimes$ ground-glass opacities were found in 57 patients, among which 11 patient showed a diffuse distribution of lesions in both lungs, another 4 in a single lung lobe, and 42 patients had lesions in multiple lobular segments. The lesions were found in more than two lung lobes or segments, usually with non-uniform density. There were more lesions in the lower lobes in both lungs. The lesions typically had an arc-shaped distribution in the peripheral lung field adjacent to the subpleural region. $\otimes$ Pulmonary interstitial shadows and fibrous stripes were found in 37 patients (including 2 patients who also had groundglass opacities) , among whom 11 cases had interlobular septal thickening, presenting as pulmonary interstitial or interlobular septal thickening adjacent to the pleura; 6 cases had subpleural linear shadows, presenting as an arched shadow parallel to the pleura within $1 \mathrm{~cm}$ of the subpleural region; $3 \mathrm{cases}$ had

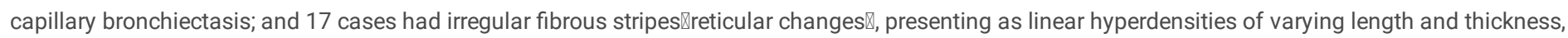
which were generally found in the peripheral lung field and the lung field adjacent to the diaphragm.

3.CT features upon the second reexamination: 94 recovered patients returned to the hospital for the second reexamination at 43-88 days postdischarge, 17 of whom had normal CT features of the lungs. All of the ground-glass opacities showed absorption to varying degrees. The ground-glass opacities were well absorbed in 39 patients, with continued decrease in extent of opacification and shrinkage of the lesions. Residual interstitial shadows were still present in 35 patients, where the irregular fibrous stripes $\llbracket$ reticular changes, interlobular septal thickening, and subpleural linear shadows had not significantly improved.

4.CT features upon third examination: 77 recovered patients returned to the hospital for the third reexamination at 112-260 days post-discharge, 20 of whom had normal CT features of the lungs. All of the ground-glass opacities showed absorption substantially. Where residual ground-glass opacities in 13 cases $囚$ the irregular fibrous stripes $\llbracket$ reticular changes in 25 cases, interlobular septal thickening in 12 cases, and subpleural linear shadows in 6 cases.

5.CT features upon fourth examination: 57 recovered patients returned to the hospital for the fourth reexamination at 195-465 days post-discharge, 6 of whom had normal CT features of the lungs. All of the ground-glass opacities showed absorption substantially. Where slightest residual ground-glass opacities in 1 case $₫$ the irregular fibrous stripes $\llbracket$ reticular changes in 31 cases, interlobular septal thickening in 10 cases, and subpleural linear shadows in 5 cases. The FEV $1.0 \%$ of the two groups were different in the first three reexamination. The pulmonary function of the mild group was better than that of the severe group,

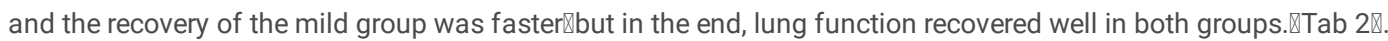

\section{Discussion}

The prognosis and outcome of recovered COVID-19 patients are of great concern. Reexamination by CT scan is necessary to assess pulmonary lesions in these patients. The lung function of a small number of COVID-19 patients has been shown to be severely impaired. The CT manifestations of these recovered COVID-19 patients are closely related to their activities of daily living $8,9,10$.

The residual lesions found by pulmonary CT scan in the recovered COVID-19 patients usually include ground-glass opacities and interstitial shadows (interlobular septal thickening, subpleural linear shadow and irregular stripe shadows $\llbracket$ reticular changes). Interstitial shadows do not occur alone, but generally following the ground-glass opacities. In our study, the ground-glass opacities were gradually absorbed and reduced in density. This was especially true when the lesions affected less than 2 lobes or when the lesions were located in less than 2 pulmonary segments and had lower density. The fibrous stripe shadows and subpleural linear shadows can also partially absorbed. However, the absorption is less likely for stripe shadows with a higher density. In some patients, the lesions remained hardly changed on repeat CT scan three months later. Antonio GE et al. Reported ${ }^{11}$ that stripe shadows in the pulmonary parenchyma, irregular interface sign, and traction bronchiectasis were the signs of fibrosis, which in turn were closely related to the patients' age and gender. That is, residual intrapulmonary fibrotic lesions are more likely to be found in elderly males. According to the autopsy of COVID-19 patients, the early lesions included exfoliation of bronchiolar epithelium, cilia shedding, squamous metaplasia and atypically enlarged alveolar cells ${ }^{12}$. At the early stage of COVID-19, some patients with fever did not present with apparent exudative lesions upon chest X-ray or CT. The pulmonary shadows did not occur until 3-7 days later. This feature agrees with the fact that the early pathological lesions primarily occur in the bronchi without abnormal findings in the lungs. COVID-19 infection caused stimulation to the epithelial cells at the early stage, leading to intrapulmonary cell proliferation and squamous metaplasia. Patients with a course of disease shorter than ten days had hyaline membrane formation in the lungs, alveolar cell proliferation, and edema; those with a longer course of disease presented with diffuse alveolar damage ${ }^{13,14}$. Nicholls JM further divided the lesions into exudative, proliferative, and fibrotic stages ${ }^{15}$. Johkoh T et al. believed that the ground-glass opacities in the exudative stage of infectious pneumonia reflected the edema in the alveolar septum and the formation of the hyaline membrane in the alveolar wall. At the proliferative and fibrotic stages(15-30 days), alveolar and interstitial proliferation and fibrosis were observed.Therefore, the pathological changes accorded with the natural follow-up radiological results ${ }^{16,17}$.

During the follow-up period of 22-465 days, we found that the pulmonary function of convalescent patients with COVID-19 was damaged to some extent after discharge, which was related to residual ground glass opacity $\llbracket$ reticular changes and fibrosis of CT. Especially in severe patients with ARDS and older male patients during hospitalization, pulmonary diffusion function decreased significantly, It is reported that the convalescent lesions of COVID-19 mainly exist in the alveolar wall, affecting the gas exchange through the alveolar capillary membrane, leading to the decline of diffusion function. In the early stage of rehabilitation in our study, the lung function of $89.83 \%$ of the convalescent patients recovered with the prolongation of the reexamination time. Only 12 patients' diffusion function did not fully recover to the normal level. At present, we continue to follow up the pulmonary function of the patients. Through the follow-up of chest CT after discharge, HRCT findings of covid-19 rehabilitation patients are closely related to their clinical manifestations, laboratory examination and pulmonary function. With the reduction of ground glass shadow $\llbracket$ reticular changes and fiber strips on $\mathrm{CT}$, residual lung lesions and lung function can be gradually improved, but interstitial shadow absorption is relatively slow.At the same time, we found that $\$ given the rampant infectivity of covid-19 virus, the psychological pressure of rehabilitation patients is tremendous. With the substantial improvement of CT changes, it also accelerates the recovery of mental health of patients and makes them better integrate into normal social life ${ }^{18,19,20}$ 


\section{Conclusion}

Reexamination by HRCT may reveal ground-glass opacities and other residual lesions in recovered COVID-19 patients. As time progresses, the intrapulmonary lesions gradually improve or even disappear; however, the pulmonary interstitial shadows $\square$ reticular changes and fibrous stripes have been noted to be absorbed more slowly.

\section{Declarations}

- Ethical Approval and Consent to participate囚The experimental protocol was established, according to the ethical guidelines of the Helsinki Declaration and was approved by the Human Ethics Committee of Xiangyang First People's Hospital affiliated to Hubei Medical University (Issue No. S201 [2020]). Written informed consent was obtained from individual or guardian participants.

- Consent for publication $₫$ Yes, we all agree.

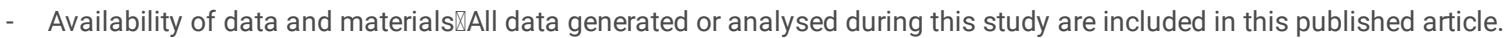

- Competing interests $\varangle$ Disclosure of interests $\otimes T h e$ authors declare no conflicts of interest.

- Funding $\llbracket$ Not applicable.

- Authors' contributions $₫$ Peng Ann conceived and drafted the manuscript. All of the authors contributed to the literature review, revised the manuscript critically for important intellectual content, Peng Ann approved the final version to be published and agreed to act as guarantors of the work.

- Acknowledgements $\bigotimes$ The authors gratefully acknowledge Jing Liu and Kai Guo for assistance with translating references. The study was funded by the Agency for Integrated Care, Red Cross and Development Board and Ministry of Hubei Health. The authors would like to thank the caregivers for taking time to participate in the research study and for generously sharing their experiences and medical informations.

- Authors' information『PENG ANN囚Department of Radiology, Xiangyang No. 1 People's Hospital Affiliated to Hubei University of Medicine. Hubei ,441000,China.Department of Radiology, The Affiliated Hospital of Nanjing University of Chinese Medicine,Jiangsu Province Hospital of Chinese medicine,The First Clinical Medical College, 155 Hanzhong Road, Nanjing 210029, Jiangsu Province, China.

\section{References}

1. World Health Organization. WHO Coronavirus Disease (COVID-19) Dashboard. Available at: (https://www.who.int. Accessed on xxday xxmonth xxyear.

2. World Health Organization. Coronavirus disease (COVID-19) outbreak (https://www.who.int. opens in new tab).

3. Zhou JC, Hui TCH, Tan CH, Khoo HW, et al. Chest Radiography in Coronavirus Disease 2019 (COVID-19): Correlation with Clinical Course. Ann Acad Med Singap 2020;49:456-61.

4. Ai T, Yang Z, Hou H, et al. Correlation of Chest CT and RT-PCR Testing for Coronavirus Disease 2019 (COVID-19) in China: A Report of 1014 Cases. Radiology 2020;296:E32-E40. doi: 10.1148/radiol.2020200642. Epub 2020 Feb 26. PMID: 32101510; PMCID: PMC7233399.

5. Clinical management of severe acute respiratory infection when novel coronavirus (nCoV) infection is suspected冈https://www.who.int/docs/defaultsource/coronaviruse/clinical-management-of-novel-cov.pdf囚.

6. Chew SY, Lee YS, Ghimiray D, Tan CK, Chua GSW. Characteristics and Outcomes of COVID-19 Patients with Respiratory Failure Admitted to a "Pandemic Ready" Intensive Care Unit - Lessons from Singapore. Ann Acad Med Singap 2020; 49: 434-48.

7. General Office of the National Health and Health Commission. Notice on Issuing a New Coronavirus Pneumonia Diagnosis and Treatment Plan (Trial Implementation of Revised Fifth Edition). National Health Office Medical Letter [2020] No. 117. 2020.

http://www.nhc.gov.cn/yzygj/s7653p/202002/3b09b894ac9b4204a79db5b8912d4440.shtml

8. An P, Wood BJ, Li W, Zhang M, Ye Y. Postpartum exacerbation of antenatal COVID-19 pneumonia in 3 women. CMAJ. 2020 Jun 1;192(22):E603-E606. doi: 10.1503/cmaj.200553. Epub 2020 May 6. PMID: 32376643; PMCID: PMC7272195.

9. Carfi A, Bernabei R, Landi F; Gemelli Against COVID-19 Post-Acute Care Study Group. Persistent Symptoms in Patients After Acute COVID-19. JAMA. 2020 Aug 11;324(6):603-605. doi: 10.1001/jama.2020.12603. PMID: 32644129; PMCID: PMC7349096.

10. Goh KJ, Choong MC, Cheong EH, Kalimuddin S, Duu Wen S, Phua GC, Chan KS, Haja Mohideen S. Rapid Progression to Acute Respiratory Distress Syndrome: Review of Current Understanding of Critical Illness from COVID-19 Infection. Ann Acad Med Singap 2020 Mar 16;49(3):108-118.

11. Antonio GE, Wong KT, Hui DS, et al. Thin-section CT in patients with severe acute respiratory syndrome following hospital discharge: preliminary experience. Radiology. 2003;228(3):810-815. doi:10.1148/radiol.2283030726

12. Xu Z, Shi L, Wang Y, Zhang J, Huang L, Zhang C, et al. Pathological findings of COVID-19 associated with acute respiratory distress syndrome. Lancet Respir Med. 2020 Apr;8(4):420-422. doi: 10.1016/S2213-2600(20)30076-X. Epub 2020 Feb 18. Erratum in: Lancet Respir Med. 2020 Feb 25;: PMID: 32085846 ; PMCID: PMC7164771.

13. Tan CS, Yeoh SF, Long CM. COVID-19: Critical Role of Angiotensin 1-7 in ACE2 Modulation. Ann Acad Med Singapore. 2020 Jun;49(6):398-400. PMID: 32712639 
14. Bhandari S, Sharma S, Bhargava A, Keswani P, Sharma R, Shekhawat A. Inflammatory Markers in COVID-19. Ann Acad Med Singapore. 2020 Jun;49(6):393-397. PMID: 32712638.

15. Nicholls JM, Poon LL, Lee KC, Ng WF, Lai ST, Leung CY, et al. Lung pathology of fatal severe acute respiratory syndrome. Lancet. 2003 May 24;361(9371):1773-8. doi: 10.1016/s0140-6736(03)13413-7. PMID: 12781536; PMCID: PMC7112492.

16. Johkoh T, Müller NL, Taniguchi H, Kondoh Y, Akira M, Ichikado K, et al. Acute interstitial pneumonia: thin-section CT findings in 36 patients. Radiology. 1999 Jun;211(3):859-63. doi: 10.1148/radiology.211.3.r99jn04859. PMID: 10352616.

17. Goh KJ, Choong MC, Cheong EH, Kalimuddin S, Duu Wen S, Phua GC, et al. Rapid Progression to Acute Respiratory Distress Syndrome: Review of Current Understanding of Critical Illness from COVID-19 Infection. Ann Acad Med Singapore. 2020 Mar 16;49(3):108-118. PMID: 32200400.

18. Wang Y, Dong C, Hu Y, et al. Temporal Changes of CT Findings in 90 Patients with COVID-19 Pneumonia: A Longitudinal Study. Radiology. 2020;296(2):E55-E64. doi:10.1148/radiol.2020200843

19. Liu D, Zhang W, Pan F, et al. The pulmonary sequalae in discharged patients with COVID-19: a short-term observational study. Respir Res. $2020 ; 21$ (1):125. Published 2020 May 24. doi:10.1186/s12931-020-01385-1.

20. Pan F, Ye T, Sun P, et al. Time Course of Lung Changes at Chest CT during Recovery from Coronavirus Disease 2019 (COVID-19). Radiology. 2020;295(3):715-721. doi:10.1148/radiol.2020200370.

\section{Tables}

Table 1 COVID-19 pneumonia diagnostic criteria for mild and severe cases formulated by WHO

Mild Patients uncomplicated upper respiratory tract viral infection may have non-specific symptoms such as fever, fatigue, cough (with or without cases sputum production), anorexia, malaise, muscle pain, sore throat, dyspnea, nasal congestion, or headache. Rarely, patients may also present with diarrhoea, nausea, and vomiting. The elderly and immunosuppressed may present with atypical symptoms. Symptoms due to physiologic adaptations of pregnancy or adverse pregnancy events, such as dyspnea, fever, Gl-symptoms or fatigue, may overlap with COVID-19 symptoms.

Severe Adolescent or adult: fever or suspected respiratory infection, plus one of the following: respiratory rate $>30$ breaths/min; severe respiratory cases distress; or SpO2 $\leq 93 \%$ on room air. Child with cough or difficulty in breathing, plus at least one of the following: central cyanosis or SpO2 < $90 \%$; severe respiratory distress (e.g. grunting, very severe chest indrawing); signs of pneumonia with a general danger sign: inability to breastfeed or drink, lethargy or unconsciousness, or convulsions. Other signs of pneumonia may be present: chest indrawing, fast breathing (in breaths/min): $<2$ months: $\geq 60 ; 2-11$ months: $\geq 50 ; 1-5$ years: $\geq 40$. While the diagnosis is made on clinical grounds; chest imaging may identify or exclude some pulmonary complications.

Table 2 Comparison of high resolution CT findings at two serial intermittent re-examinations in convalescent patients after COVID-19

\begin{tabular}{|c|c|c|c|c|c|c|c|c|c|c|c|}
\hline \multirow[t]{2}{*}{ Findings } & \multicolumn{3}{|c|}{ First CT examination $(n=118)$} & \multicolumn{3}{|c|}{ Second CT examination $(n=94)$} & \multicolumn{3}{|c|}{ Third CT examination $(n=77)$} & \multicolumn{2}{|c|}{ Fourth CT examir } \\
\hline & $\begin{array}{l}\text { mild group } \\
(n=71)\end{array}$ & $\begin{array}{l}\text { sever } \\
\text { group } \\
(\mathrm{n}=47)\end{array}$ & $\begin{array}{l}\text { total } \\
(n=118)\end{array}$ & $\begin{array}{l}\text { mild group } \\
(n=48)\end{array}$ & $\begin{array}{l}\text { sever } \\
\text { group } \\
(n=46)\end{array}$ & $\begin{array}{l}\text { total } \\
(n=94)\end{array}$ & $\begin{array}{l}\text { mild } \\
\text { group } \\
(n=36)\end{array}$ & $\begin{array}{l}\text { sever } \\
\text { group } \\
(n=41)\end{array}$ & $\begin{array}{l}\text { total } \\
(n=77)\end{array}$ & $\begin{array}{l}\text { mild } \\
\text { group } \\
(n=22)\end{array}$ & $\begin{array}{l}\text { sevt } \\
\text { grol } \\
(n=?\end{array}$ \\
\hline \multicolumn{12}{|l|}{ CT findings } \\
\hline Normal & 18 & 6 & 24 & 11 & 6 & 17 & 13 & 7 & 20 & 5 & 1 \\
\hline $\begin{array}{l}\text { ground-glass } \\
\text { opacities }\end{array}$ & 40 & 17 & 57 & 25 & 14 & 39 & 8 & 5 & 13 & 1 & 3 \\
\hline Subpleural lines & 1 & 5 & 6 & 2 & 3 & 5 & 2 & 4 & 6 & 1 & 4 \\
\hline $\begin{array}{l}\text { Intralobular } \\
\text { septal } \\
\text { thickening }\end{array}$ & 4 & 7 & 11 & 2 & 7 & 9 & 3 & 9 & 12 & 2 & 8 \\
\hline $\begin{array}{l}\text { Irregular lines] } \\
\text { fibrous cord } \\
\text { (reticular } \\
\text { changes) }\end{array}$ & 6 & 11 & 17 & 7 & 14 & 21 & 10 & 15 & 25 & 13 & 18 \\
\hline Bronchiolectasis & 2 & 1 & 3 & 1 & 2 & 3 & 0 & 1 & 1 & 0 & 1 \\
\hline $\mathrm{FEV}_{1} \square$ & $79.39 \pm 3.47$ & $71.27 \pm 2.37$ & & $80.91 \pm 2.51$ & $76.87 \pm 4.68$ & & $84.2 \pm 1.78$ & $79.18 \pm 2.59$ & & $85.2 \pm 2.78$ & 84.1 \\
\hline T/P value & $\mathrm{T}=8.948$ & $P \rrbracket 0.001$ & & $\mathrm{~T}=3.489$ & Pヌ0.001 & & $\mathrm{T}=6.241$ & $P \otimes 0.001$ & & $\mathrm{~T}=1.321$ & $\mathrm{P} \otimes 0$ \\
\hline
\end{tabular}

\#The initial 24 patients who had normal CT exam at the 1 st sitting were excluded from the 2nd imaging.

And the 17 patients who had normal CT exam at the 2st sitting were excluded from the 3 nd imaging.

\section{Figures}




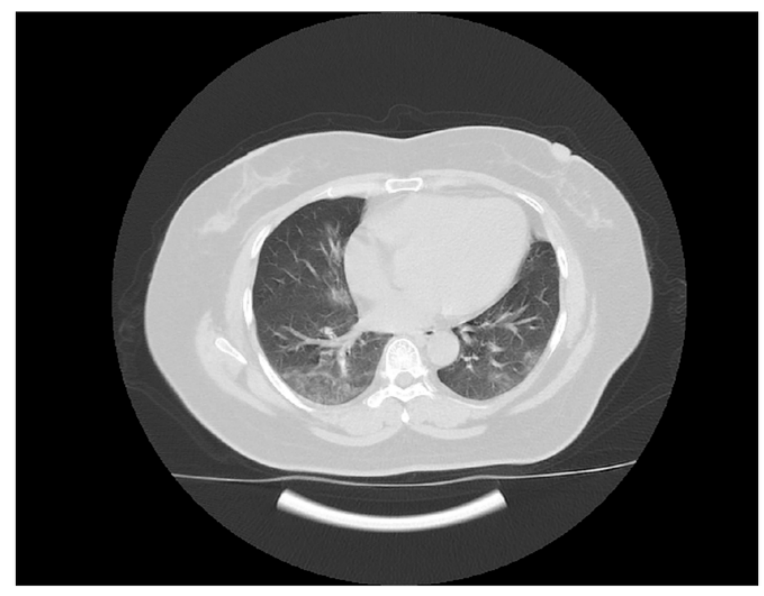

A

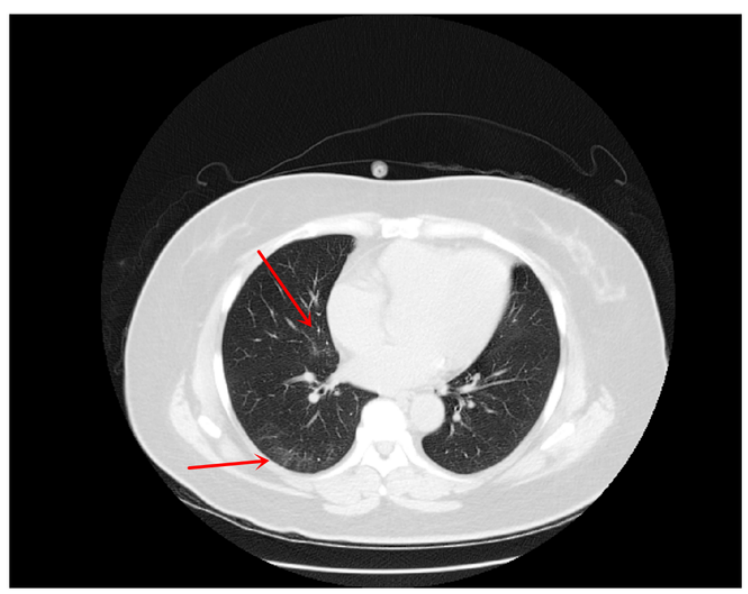

B

Figure 1

A, a 50-year-old female patient; on February 3rd, 2020, the non-contrast enhanced CT scan revealed lumpy and patchy ground-glass opacities in both lungs. B, 89 days later after discharge, the non-contrast enhanced CT scan revealed ground-glass opacities in both lower lobes and near the pleura『arrow $\mathbb{}$ CT changes are improved囚. 


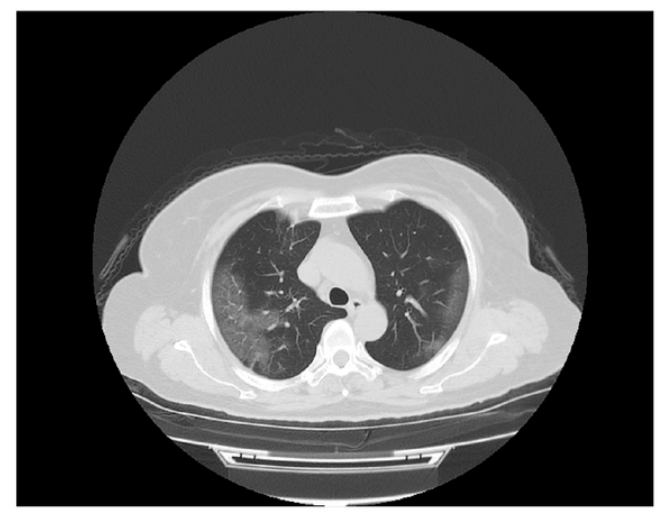

A

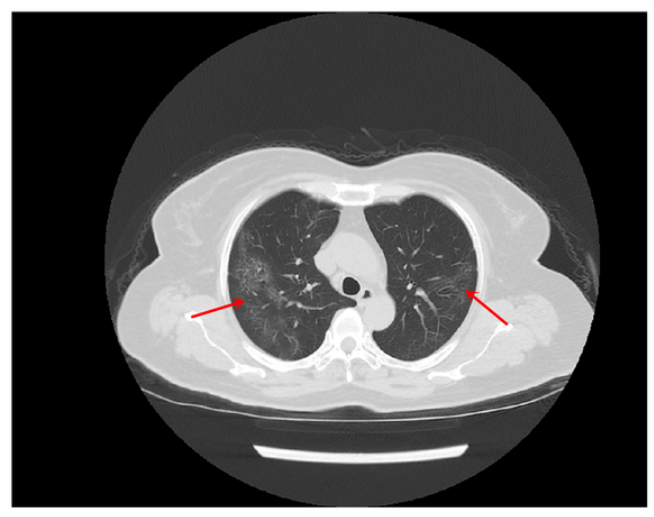

B

Figure 2

A, a 60-year-old male patient; on January 30th, 2020, the non-contrast enhanced CT scan revealed ground-glass opacities in both lungs. B, 62 days later after discharge, the non-contrast enhanced CT scan revealed ground-glass opacities and interlobular septal thickening in both upper lobes and near the pleura \arrow凶CT changes are improved囚. 


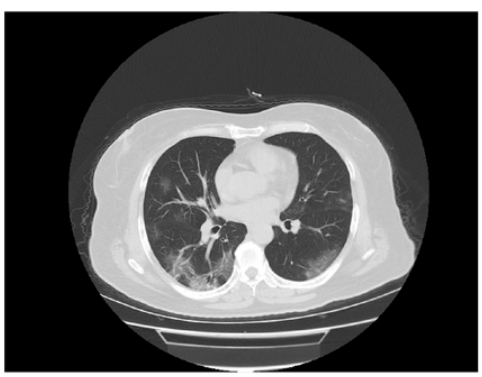

A

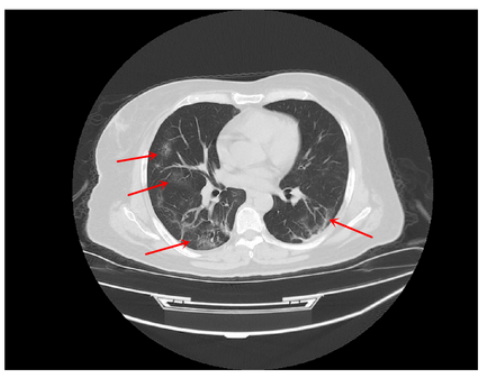

B

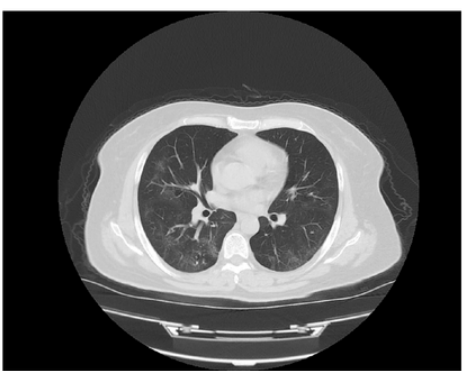

c

\section{Figure 3}

A, a 62-year-old female patient; on February 4th, 2020, the non-contrast enhanced CT scan revealed multiple flocculent and patchy ground-glass opacities in both lungs. B, 53 days later after discharge, the non-contrast enhanced CT scan revealed ground-glass opacities and fibrous stripes in both lower lobes and

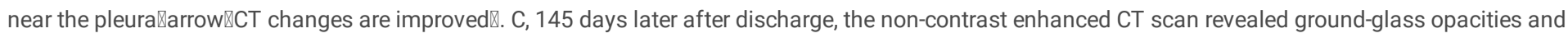
fibrous stripes were improved substantially. 